\title{
THE FOURIER TRANSFORMS OF LIPSCHITZ FUNCTIONS ON CERTAIN DOMAINS
}

\author{
M.S. YOUNIS \\ Department of Mathematics \\ Yarmouk University \\ Irbid, JORDAN
}

(Received December 13, 1995)

\begin{abstract}
The Fourier transforms of certain Lipschitz functions are discussed and compared with the Hankel transforms of these functions and with their Fourier transforms on the Euclidean Cartan Motion group $M(n), n \geq 2$
\end{abstract}

KEY WORDS AND PHRASES: Fourier transforms, Fourier series, Lipschitz functions, absolute convergence

1991 AMS SUBJECT CLASSIFICATION CODES: Primary 42B05, 42B10; Secondary 43A30, 22E30

\section{INTRODUCTION}

The famous Bernstein's Theorem states that if the function $f(x)$ belongs to the Lipschitz class $\operatorname{Lip}(\alpha), \alpha>\frac{1}{2}$ on the circle group $T=[0,2 \pi]$, then its Fourier series is absolutely convergent (see [10], Vol 1, p. 240). This theorem has been extended and generalized in various directions. The purpose of the present work is to study the Fourier transforms of various Lipschitz functions and to compare some of the results obtained here with the Hankel transforms of these functions and with their Fourier transforms on the group $M(n)$.

The main feature of our treatment in this work is a change of variables from Cartesian to polar coordinates This appears to be the binding thread which relates some results concerning the Fourier transforms of certain Lipschitz functions with the Hankel (Fourier-Bessal) transforms of these functions

Our paper is organized as follows. In section 2 the necessary definitions and notations are given. In section 3 we study the Fourier transforms (coefficients) of various Lip-classes by changing from Cartesian to polar coordinates with special emphasis on radial functions. Section 4 contains a brief comparison between some of our results with those obtained for the Hankel transforms of certain Lipschitz functions and with the Fourier transforms of these functions when defined on $M(n)$

\section{DEFINITIONS AND NOTATIONS}

This section will be brief since most of our definitions and notations are standard In the sequel $T, T^{n}, R$ and $R^{n}$ denote the circle group, the $n$-dimensional torus, the real line, and the $n$-dimensional Euclidean space respectively. The function $f$ of several variables is written as $f(x)=f\left(x_{1}, x_{2}, \ldots, x_{n}\right)$ and $(x . y)$ stands for the inner product

$$
(x . y)=x_{1} y_{1}+x_{2} y_{2}+, \ldots,+x_{n} y_{n}
$$


The $L^{p}$-space consists of the equivalence classes of functions whose $p$-th power is Lebesgue-integrable with the usual symbol $\|\cdot\|_{p}$ for the $L^{p}$-norm The $r$-th difference in step $h$ for $f(x)$ is given by

$$
\triangle_{h}^{r} f(x)=\sum_{i=0}^{r}(-)^{r-2}\left(\begin{array}{l}
r \\
i
\end{array}\right) f(x+i h) .
$$

For a nonnegative increasing function $w(x)$ the weighted $L^{p}$-space is defined such that

$$
\int|f|^{p} w d x<\infty
$$

Our definitions are stated for functions of two variables for the sake of brevity Their extension to functions in $R^{n}$ is quite obvious.

DEFINITION 2.1. Let $f(x)=f\left(x_{1}, x_{2}\right)$ belong to $L^{p}\left(R^{2}\right)$ We say that $f$ is in the Lipschitz space $\operatorname{Lip}\left(\alpha_{1}, \alpha_{2}, p\right)$ if

$$
\left\|\begin{array}{l}
f\left(x_{1}+h_{1}, x_{2}+h_{2}\right)-f\left(x_{1}, x_{2}+h_{2}\right) \\
-f\left(x_{1}+h_{1}, x_{2}\right)+f\left(x_{1}, x_{2}\right)
\end{array}\right\|_{p}=O\left(h_{1}^{\alpha_{1}} h_{2}^{\alpha_{2}}\right)
$$

as $h_{1}, h_{2}$ tend to zero, $1 \leq p<\infty, 0<\alpha_{1}, \alpha_{2} \leq 1$. Another definition which will be frequently used throughout is the following

DEFINITION 2.2. The function $f\left(x_{1}, x_{2}\right)$ belongs to $\operatorname{Lip}\left(\alpha_{1}, \alpha_{2}, p\right)$ if

$$
\left\|f\left(x_{1}+h_{1}, x_{2}+h_{2}\right)-f\left(x_{1}, x_{2}\right)\right\|_{p}=O\left(h_{1}^{\alpha_{1}}+h_{2}^{\alpha_{2}}\right)
$$

where $\alpha_{1}, \alpha_{2}, h_{1}, h_{2}$, and $p$ are the same as in (2.1) The function $f(x)$ is called radial if

$$
f(x)=g(r)=g\left(\sqrt{x_{1}^{2}+x_{2}^{2}}\right) .
$$

The Fourier transform $\widehat{f}(y)$ of $f(x)$ is defined to be

$$
\begin{aligned}
\widehat{f} & =f\left(y_{1}, y_{2}\right)=\int f(x) e^{-z(x \cdot y)} d x \\
& =\iint f\left(x_{1}, x_{2}\right) e^{-z\left(x_{1} y_{1}+x_{2} y_{2}\right)} d x_{1} d x_{2} .
\end{aligned}
$$

Integration is taken over the plane $R^{2}$.

\section{FOURIER TRANSFORMS OF LIPSCHITZ FUNCTIONS}

In this section we examine the effect of the smoothness conditions (2.1) and (2.2) on the Fourier transform $\hat{f}$ of $f(x)$ by changing to polar coordinates. The merit of this approach appears in simplifying the arguments in the proof and in unifying the version of theorems proved in different areas of Fourier analysis For brevity, the term "transform" stands here interchangeably for the Fourier transform and the Fourier coefficient unless any otherwise is explicitly mentioned. We start with the following theorem.

THEOREM 3.1. Let $f(x)$ belong to $L^{p}\left(R^{2}\right), 1<p \leq 2$ such that (2.2) is satisfied. Then

$$
\begin{gathered}
\sum_{n=1}^{\infty} \int_{0}^{\infty}|\widehat{f}(u, n)|^{\beta} d u<\infty \\
\text { for } \frac{p}{(p+\alpha p / 2-1)}<\beta \leq q=\frac{p}{(p-1)}, \quad \alpha=\min \left(\alpha_{1}, \alpha_{2}\right) \text {. }
\end{gathered}
$$

PROOF. By changing to polar coordinates, with $h^{2}=h_{1}^{2}+h_{2}^{2}$ one finds that $f\left(x_{1}+h_{1}, x_{2}+h_{2}\right)-$ $f\left(x_{1}, x_{2}\right)$ is equal to

$$
f((r+h) \cos \theta,(r+h) \sin \theta)-f(r \cos \theta, r \sin \theta))=g(r, \theta)
$$


for instance, $\widehat{f}$ is then written as $\widehat{f}(u, n)$, where $u \in R^{+}=[0, \infty)$, and $n$ is an integer Thus the transform of $g$ is given by

$$
\widehat{g}(r, \theta)=\left(e^{-\imath u h}-1\right) \widehat{f}(u, n) .
$$

Applying the Hausdorff-Young inequality and taking into account that $h_{1}, h_{2}=O(h), \alpha=\min \left(\alpha_{1}, \alpha_{2}\right)$ we obtain

$$
\sum \int|\sin u h \widehat{f}|^{q} d u=O\left(h^{\alpha q}\right)
$$

If $0<u<\frac{1}{h}$, then $|u h|<A|\sin u h|, A$ being constant and therefore

$$
\sum_{1}^{N} \int_{1}^{X}|u \widehat{f}|^{q} d u=O\left(X^{(1-\alpha) q}\right)
$$

$X=\frac{1}{h}$ For $\beta \leq q$ and argument similar to that in Titchmarsh ([4], Theorem 84, $\mathrm{p} 115$ ) yields

$$
\sum_{1}^{N} \int_{1}^{X}|\widehat{f}|^{\beta} d u=O\left(X^{-\alpha \beta+1-\beta / q}\right)\left(N^{1-\beta / q}\right) .
$$

Since $N$ and $X$ are of the same order of magnitude near infinity, hence $\left(\begin{array}{ll}3 & 1\end{array}\right)$ can be written as

$$
\sum_{1}^{[X]} \int_{1}^{X}|\widehat{f}|^{\beta} d u=O\left(X^{2-\alpha \beta-2 \beta+2 \beta / p}\right) .
$$

This quantity is bounded near infinity if $\frac{p}{(p+\alpha p / 2-1)}<\beta$, and the proof is complete. Anyone who is familiar with the proof of this theorem in the ordinary Cartesian form would see the brevity and compactness of the proof given here (see [7] and [8] for example). For functions in $L^{p}\left(R^{n}\right)$ the theorem holds if $\frac{p}{(p+\alpha p / n-1)}<\beta \leq q$, where the left side of (3.1) is to be replaced by another containing one integral sign for the continuous variable $u$ and $(n-1)$ summations for the discrete variables of $\widehat{f}$ The proof is quite direct and is therefore omitted. We notice that if (2.1) is applied instead of (2 2), then the second difference $\Delta_{h_{1}}\left(\Delta_{h_{2}} f\right)=\triangle_{h_{2}}\left(\triangle_{h_{1}} f\right)$ is equivalent to the quantity $\triangle_{h}\left(\triangle_{h} f\right)=\triangle_{h}^{2} f(r \cos \theta, r \sin \theta)=\triangle_{h}^{2} g(r, \theta)$, where $h_{1}^{\alpha_{1}} h_{2}^{\alpha_{2}}=O\left(h^{\alpha_{1}+\alpha_{2}}\right)$. At this point one is at liberty either to keep the choice $0<\alpha_{1}, \alpha_{2} \leq 1$, or to take - equivalently $-0 \leq \alpha \leq 2$. Thus we state the following theorem.

THEOREM 3.2. Let the hypothesis of Theorem 3.1 hold such that $(2.1)$ is satisfied Then the conclusion of the theorem holds if $\frac{p}{\left(p+\left(\alpha_{1}+\alpha_{2}\right) p / 2-1\right)}<\beta$, or - equivalently - if $\frac{p}{(p+\alpha p / 2-1)}<\beta$.

PROOF. In polar coordinates (2.1) takes the form

$$
\int_{0}^{\infty} \int_{0}^{2 \pi}\left|\triangle_{h}^{2} f(r \cos \theta, r \sin \theta)\right|^{p} r d r d \theta=O\left(h^{\alpha p}\right)
$$

and consequently

$$
\begin{gathered}
\sum \int\left|\left(e^{-\imath u h}-1\right)^{2} \widehat{f}(u, n)\right|^{q} d u=O\left(h^{\alpha q}\right) \\
\sum_{1}^{N} \int_{1}^{X}|u h \widehat{f}|^{q} d u=O\left(h^{\alpha q}\right)
\end{gathered}
$$


and finally for $\beta \leq q$

$$
\sum_{1}^{[X]} \int_{1}^{X}|\widehat{f}|^{\beta} d u=O\left(X^{2-\alpha \beta-2 \beta+2 \beta / p}\right)
$$

$[X]$ being the integral part of $X$. The rest of the proof is obvious and the theorem is proved It is clear that the condition (2.1) is stronger than that in (2.2); this is reflected on the range of $\beta$ in the two theorems For the proof and the conclusion of Theorem 3.2 in Cartesian coordinates the interested reader may consult one of the references [7], [8], for example. It should be observed that for functions in $L^{p}\left(T^{n}\right)$ one is dealing with the Fourier coefficients proper, so the integral sign disappears and the product of $n$-summations takes place It might be convenient to notice that the way it is stated here, the condition $\frac{p}{(p+\alpha p / n-1)}<\beta$ demonstrates more obviously the relation between the smoothness exponent $\alpha$ and the space dimension $n$ in case of functions of several variables.

REMARK 3.3. We mention here that Theorem 3.2 was proved for functions in $L^{p}\left(R^{n}\right)$ and in $L^{p}\left(T^{n}\right)$ by rather different methods from the one just carried throughout One may refer to [5] (satz. 1) and to [9] (Theorem 2.15, p. 39).

\section{RADIAL FUNCTIONS AND HANKEL TRANSFORMS}

Our aim here is to carry the above analysis for radial functions. The importance of this approach lies in the fact that it links up several ideas such as the weighted Lipschitz spaces, the Hankel transforms and the transforms on the group $M(n)$ In this case (2.1) and (2.2) are written as

$$
\int_{0}^{\infty}\left|\triangle_{h}^{2} g(r)\right|^{p} r d r=O\left(h^{\left(\alpha_{1}+\alpha_{2}\right) p}\right)
$$

and

$$
\int_{0}^{\infty}\left|\triangle_{h} g(r)\right|^{p} r d r=O\left(h^{\alpha_{1}}+h^{\alpha_{2}}\right)^{p}
$$

If $f(x)$ is a radial function of $n$ variables, then (4 1) and (4.2) are to be slightly modified, the second difference in (4.1) is replaced with the $n$-th difference and $r^{n-1}$ appears in both estimates. It is well known (see [1], p. 69) that the Fourier transform of the radial function $g(r)$ is a radial function given by

$$
\widehat{g}(u)=\frac{(2 \pi)^{n / 2}}{u^{(n-2) / 2}} \int_{0}^{\infty} g(r) r^{n / 2} J_{\frac{n-2}{2}(u-r) d r} .
$$

This is just one of several definitions of the Hankel transform available in the literature. The Hankel transforms of Lipschitz functions and their Fourier transforms on $M(n)$ will be treated elsewhere Our main concern here is to prove that the Hankel transform of $(g(r+h)-g(r))$ is of the order of $\left(e^{-\imath u h}-1\right) \widehat{g}(u)$. This can be achieved as in the previous analysis without difficulty or directly by appealing to an identity of the Bessel functions (see [6], p.206, p.217) given as

$$
J_{n}(x)=\frac{1}{2 \pi} \int_{0}^{2 \pi} e^{i x \cos \theta+m \theta} d \theta .
$$

Thus one obtains

$$
J_{n}((r-h) u)=\frac{1}{2 \pi} \int_{0}^{2 \pi} e^{2(r-h) u \cos \theta+2 n \theta} d \theta
$$

In view of the boundedness of $\cos \theta$ and the compactness of $[0,2 \pi]$ this quantity is of the order of $e^{-\imath u h} J_{n}(u r)$. Thus the transform of $(g(r+h)-g(r))$ is a constant multiple of $\left(e^{-\imath u h}-1\right) \widehat{g}(u)$ minus a term majorized by $h^{\alpha}, 0<\alpha \leq 1$ This estimate is valid in our situation and can be realized without much effort The $L^{p}$-theory of the Hankel transform (see [2], Theorem 3) yields in this case 


$$
\int_{0}^{\infty} u^{n / 2}|u h \widehat{g}|^{q} d u=O\left(h^{\alpha q}\right)
$$

subsequently

$$
\int_{1}^{X}|\widehat{g}|^{q} d u=O\left(X^{-\alpha q-n / 2}\right)
$$

and for $\beta \leq q$

$$
\int_{1}^{X}|\widehat{g}|^{\beta} d u=O\left(X^{1-\alpha \beta-\beta-n \beta / 2+n \beta / 2 p+\beta / p}\right) .
$$

This is bounded for large $X$ if

$$
\frac{p}{[(\alpha+1+n / 2) p-1-n / 2]}<\beta \leq q
$$

The special cases $n=1, n=2$ are especially important since they include the results corresponding to two standard definitions of the Hankel transform with weights $w=x^{1 / 2}, w=x$ We hint that the interplay between the transforms of radial Lipschitz functions and the Hankel transforms of these functions is quite rich especially when various types of these transforms are taken into consideration. The $L^{2}$-theory is of particular significance in view of its symmetry which enables us to state our conclusions in a variety of equivalent forms (see [7], [8] for this purpose)

We turn now to a brief discussion of the Fourier transforms of Lipschitz functions defined on $M(n)$ (see [6], Chapter 4 and 11).

We focus attention on $M(2)$ first, with the polar coordinates $r, \theta, \phi$ as the group parameters (no Lie algebra is needed here) Let $g \in M(2)$. Then the Fourier transform of $f(g)$ is given by

$$
B(m, n, R)=\int_{0}^{2 \pi} \int_{0}^{\pi} \int_{0}^{\infty} f(r, \theta, \phi) e^{2 n \theta+\imath(n-m) \phi} J_{n-m}(R r) r d r d \theta d \phi
$$

In (4 5) we have suppressed a constant factor which does not affect the validity of our arguments For radial Lipschitz functions in $L^{p}(M(2))$ the previous analysis shows that

$$
\int_{0}^{\infty} R|B|^{\beta} d R<\infty
$$

if

$$
\frac{p}{(p+\alpha p / 4-1)}<\beta
$$

and

$$
\int_{0}^{\infty}|B|^{\beta} d R<\infty
$$

if $\frac{3 p}{(4 p+\alpha p-4)}<\beta$ respectively. In case of $M(n)$ where its dimension $m=\frac{n(n+1)}{2}$ one finds that $(46)$ and (4.7) are valid if

$$
\frac{p}{(p+\alpha p /(m+1)-1)}<\beta
$$

and

$$
\frac{m p}{[(\alpha+m+1) p-(m+1)]}<\beta
$$


successively This contains the results for $M(2)$ where $m=3$ It should be mentioned that the introduction of the estimates in (46) and (4.7) is not a matter of sheer repetition (they look almost equivalent) In fact they are (or at least one of them) needed in order to extract the precise analogue of Bernstein's Theorem concerning the absolute convergence of the Fourier transforms of Lipschitz functions on $M(n)$ Finally we remark that although the present treatment of the subject provided some links between certain branches of harmonic analysis, we emphasize that it would be even more valuable when applied to Lipschitz functions on more general groups For example if one adopts the Cartan decomposition of the group $\operatorname{SL}(2 \mathrm{R})$ (see [3], p. 252), then it can be easily seen that the Fourier transforms of radial functions on SL(2.R) collapse simply to a form related to the familiar Melin transforms. This remark applies to radial functions on certain types of semi-simple Lie groups as well as to radial functions on other classes of locally compact groups in general These topics will be handled in a forthcoming paper.

ACKNOWLEDGEMENT. Publication fees for this research were partially covered by Yarmouk University

\section{REFERENCES}

[1] BOCHNER, S. and CHANDRASEKHARAN, K, Fourier Transforms, Princeton University Press (1949)

[2] HERZ, C., On the mean inversion of Fourier and Hankel transforms, Proc. Nat. Acad. Sci. U.S.A., 40 (1951), 996-999

[3] SUGIURA, M., Unitary Representations and Harmonic Analysis, J Wiley, New York (1975)

[4] TITCHMARSH, E.C., Theory of Fourier Integrals, 2nd Ed., Oxford University Press (1948).

[5] TREBELS, W., Uber Absolute Summier Barkeit Von n-Dimensioneien Fourierreihen und Fourierintegralen, J. Approximation Theory, 2 (1969), 394-399.

[6] VILENKIN, N YA., Special functions and theory of group representations, Transl. Amer. Math. Soc., 22 (1968)

[7] YOUNIS, M.S., Fourier transforms of Dini-Lipschitz functions, Inter. J. Math. \& Math. Scl., 9, 2 (1986), 301-312

[8] YOUNIS, M.S., Fourier transforms of functions with symmetric differences, Acta. Math. Hung., 51 (1988), 293-299.

[9] YOUNIS, M.S., Fourier transforms of Lipschitz functions on compact groups, Ph.D. Thesis, McMaster University, Hamilton, Ontario, Canada (1974).

[10] ZYGMUND, A., Trigonometric Series, Vol. I, II, 2nd Ed., Cambridge University Press (1977). 


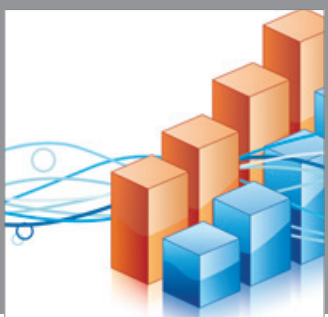

Advances in

Operations Research

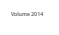

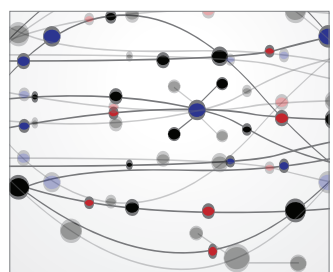

\section{The Scientific} World Journal
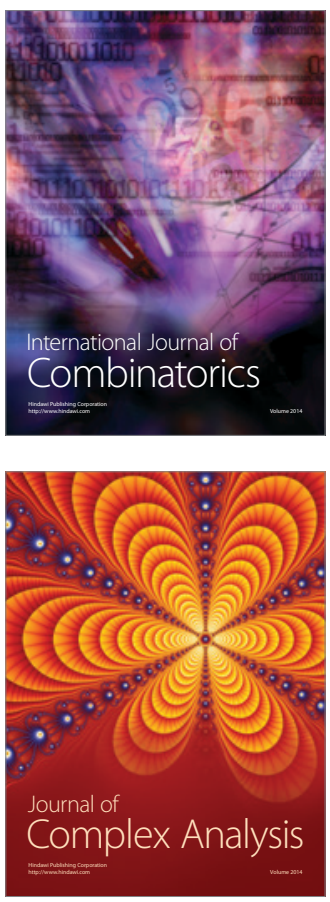

International Journal of

Mathematics and

Mathematical

Sciences
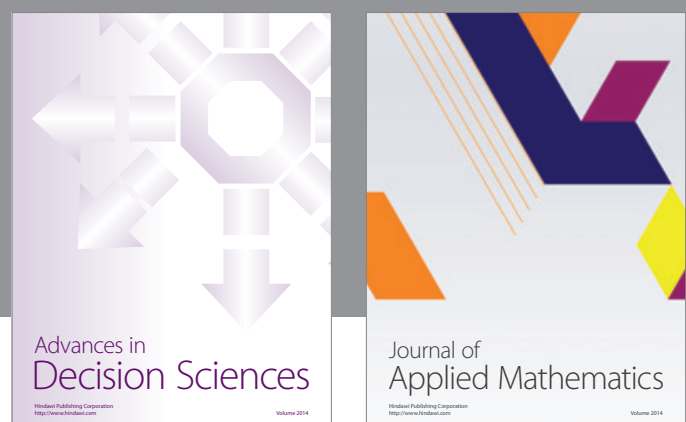

Journal of

Applied Mathematics
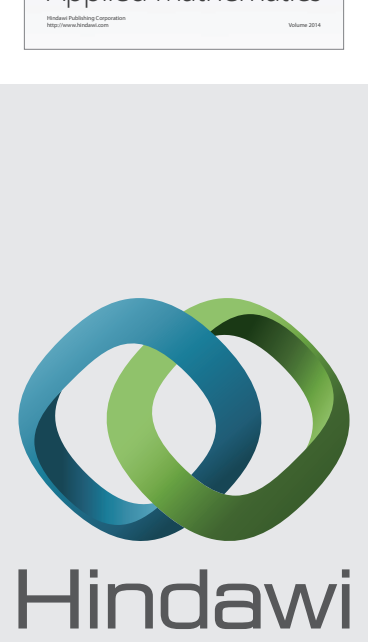

Submit your manuscripts at http://www.hindawi.com
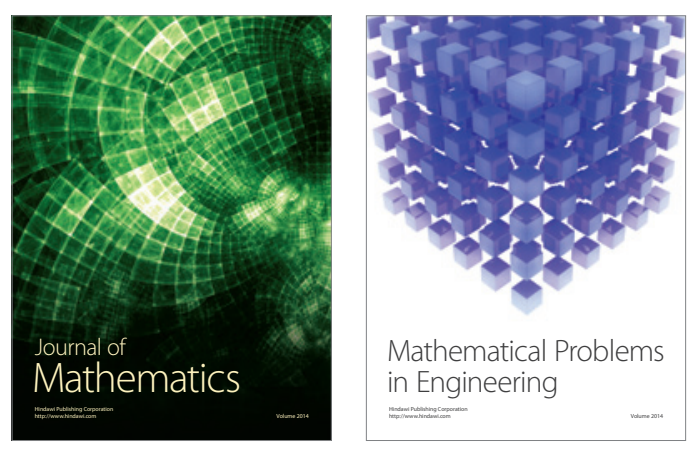

Mathematical Problems in Engineering
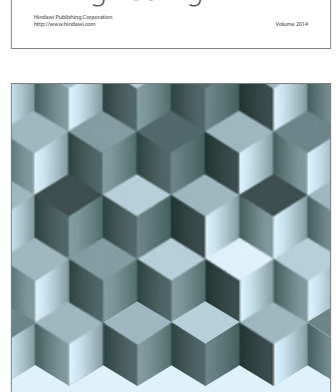

Journal of

Function Spaces
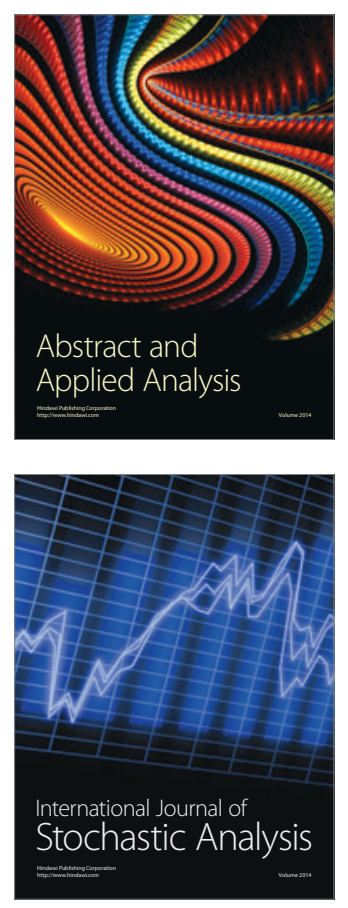

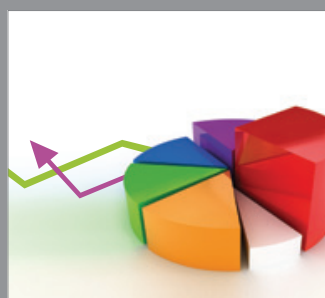

ournal of

Probability and Statistics

Promensencen
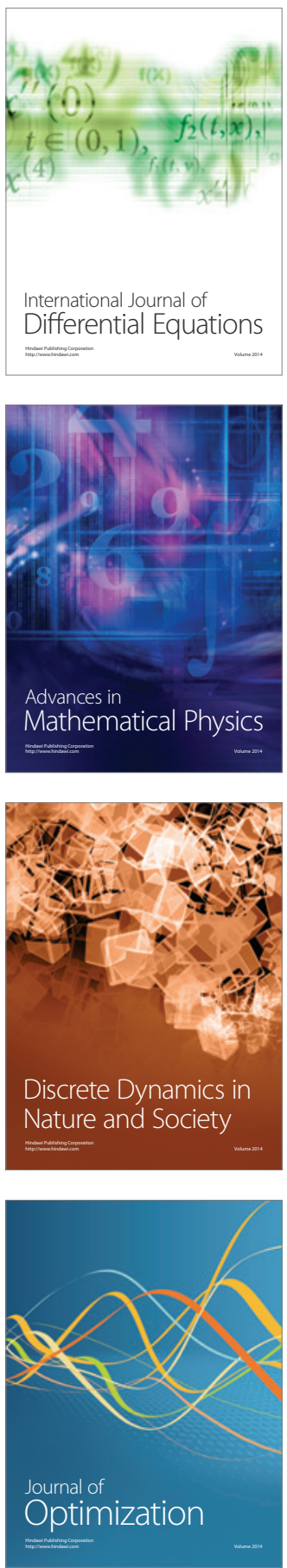